Background Para athletes may have specific mental health challenges that, together with the demands of high-level sports performance, could put them at risk for mental health disorders.

However, research in this population is limited.

Objective To investigate factors associated with mental health in South African para athletes.

Design Descriptive, cross-sectional survey.

Setting National to international level athletes competing in the 2019 National Championships.

Patients (or Participants) A total of 124 athletes (93 males; 31 females) with a mean age $26.7( \pm 9.2)$ were included in the study.

Interventions (or Assessment of Risk Factors) Demographic, medical history and sleep-related variables were included in bivariate analyses to assess their association with mental health. Between-group differences were analysed using the Mann-Whitney U or T-tests. Variables significantly associated in the bivariate analyses were included in multiple regression analyses for mental health.

Main Outcome Measurements Mental health was measured with the State/Trait Anxiety Inventory (STAI) and the Kessler Psychological Distress Scale (K-10 Questionnaire). Sleep quality, sleepiness and chronotype were measured with the Pittsburgh Sleep Quality Index (PSQI), the Epworth Sleepiness scale and Morningness-Eveningness Questionnaire (MEQ-SA).

Results The model explained $40 \%$ of the variance in mental health $(\mathrm{F}=12.04, \mathrm{p}<0.001)$ in these athletes. Compared to athletes with 'good' sleep quality, K-10 and STAI scores were significantly higher (indicating poorer mental health) in athletes with 'poor' sleep quality $(U=2.6, p<0.001 ; \mathrm{t}(116.8)=-$ $4.30, \mathrm{p}<0.001)$. 'Poor' sleep quality (B:0.8; 95\% CI 0.4 to 1.3 ), moderate to severe daytime sleepiness (B:4.2; $95 \%$ CI 1.1 to 7.3 ) intermediate (B:3.5 95\% CI 0.4 to 6.6 ) and evening chronotypes (B:12.0 95\% CI 5.0 to 19.1), the presence of allergies (B:3.9 95\% CI 0.1 to 7.6) and male gender (B:3.3 $95 \%$ CI 0.1 to 6.5 ) were most strongly associated with high STAI scores.

Conclusions This study has identified novel factors associated with poor mental health in elite para athletes. As some of these factors are modifiable, further research towards prevention strategies is warranted.

\section{SUICIDAL IDEATION AMONG ELITE ATHLETICS ATHLETES: CROSS-SECTIONAL STUDY OF ASSOCIATIONS WITH SEXUAL AND PHYSICAL ABUSE VICTIMIZATION AND PSYCHOLOGICAL RESOURCEFULNESS}

\footnotetext{
1,2Toomas Timpka, ${ }^{1,2}$ Armin Spreco, ${ }^{1,3}$ Örjan Dahlström, ${ }^{1,2}$ Jenny Jacobsson, 'Jan Kowalski, 1,2,4 Victor Bargoria, ${ }^{5}$ Margo Mountjoy, ${ }^{2}$ Carl Göran Svedin. ' Athletics Research Center, Linköping University, Linköping, Sweden; ${ }^{2}$ Department of Medical and Health Sciences, Linköping University, Linköping, Sweden; ${ }^{3}$ Department of Behavioural Sciences and Learning, Linköping University, Linköping, Sweden; ${ }^{4}$ Department of Orthopaedics and Rehabilitation, Moi University, Eldoret, Kenya; ${ }^{5}$ Department of Family Medicine, McMaster University, Hamilton, Ontario, Canada
}

\subsection{6/bjsports-2021-IOC.71}

Background Physical and sexual abuse victimization have been connected with negative effects on health long after the abuse has stopped. When athletes encounter situations of defeat and entrapment, the likelihood that suicidal ideation will emerge is increased in situations when negative motivational elements are present, for instance, at low levels of social support.
Objective To examine associations between suicidal ideation and sexual and physical abuse among active and recently retired elite Athletics (track and field) athletes.

Design Cross-sectional study.

Setting Swedish elite Athletics.

Participants Athletes $(n=402)$ who had been selected for a Swedish Athletics team for international competitions between 2011 and 2017.

Assessment of Risk Factors Lifetime experience of sexual and physical abuse, athlete personal and sociodemographic characteristics, Athletics participation, sense of coherence (SOC-13), and coping strategies (Brief Cope).

Main Outcome Measurements Suicidal ideation and 1-year period prevalence of non-sports injury.

Results 192 athletes (47.8\%) returned complete data sets. The prevalence of suicidal ideation was $15.6 \%$ (males $17.4 \%$; females 14.2\%) and the non-sports injury prevalence was 8.0\% (males 11.6\%; females 5.7\%). Suicidal ideation was among females (Nagelkerke $R^{2}=0.33$ ) associated with sexual abuse victimization (Odds ratio (OR) 5.94, 95\% Confidence interval (CI) $1.42-24.90 ; P=0.015)$ and lower sense of coherence (OR 0.90, CI 0.85-0.96; $P=0.001)$. Among males $\left(R^{2}=0.25\right)$, suicidal ideation was only associated with use of behavioural disengagement for coping (OR 1.51, CI 1.181.95; $P=0.001)$. Non-sports injury prevalence was among females $\left(R^{2}=0.23\right)$ associated with sexual abuse victimization (OR 8.61, CI $0.02-0.90 ; P=0.039$ ) and participation in an endurance event (OR 7.37, CI 1.11-48.90; $P=0.039$ ), while among males $\left(R^{2}=0.11\right)$ only having immigrant parents (OR, 5.67, CI $1.31-24.45 ; P=0.020)$ was associated with having sustained an injury outside sports.

Conclusions Given that about one out of six athletics athletes had experienced suicidal ideation, the present results warrant and can be used in suicide prevention among elite athletes.

\section{ATHLETE HEALTH MONITORING IN PARALYMPIC ATHLETES: A 52-WEEK PROSPECTIVE STUDY}

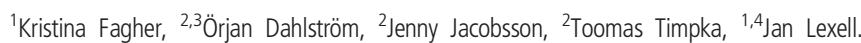
'Department of Health Sciences, Rehabilitation Medicine Research Group, Lund University, Lund, Sweden; '2Department of Medical and Health Sciences, Athletics Research Center, Linköping University, Linköping, Sweden; ${ }^{3}$ Department of Behavioural Sciences and Learning, Linköping University, Linköping, Sweden; ${ }^{4}$ Department of Neuroscience, Rehabilitation Medicine, Uppsala University, Uppsala, Sweden

\subsection{6/bjsports-2021-IOC.72}

Background Sports-related injuries and illnesses in Paralympic sport is a growing concern, but knowledge about the aetiology and risk factors is limited.

Objective To describe the annual incidence of injuries and illnesses among Paralympic athletes and to assess risk factors.

Design Prospective cohort study.

Setting Paralympic athletes

Patients (or Participants) 107 Swedish Paralympic elite athletes with vision, physical and intellectual impairments, active in 19 para sports.

Interventions (or Assessment of Risk Factors) The athletes were asked to weekly report over 52 weeks the annual incidence of injuries, illnesses and quality of health in an adapted eHealth application.

Main Outcome Measurements Time to event, incidence rate (IR) and incidence proportion (IP) and risk factors. 